\title{
Learning objects and learning designs: an integrated system for reusable, adaptive and shareable learning content
}

J. Lukasiak ${ }^{\mathrm{a}, \mathrm{b}^{*}}$, S. Agostinho ${ }^{\mathrm{a}, \mathrm{c}}, \mathrm{S}$. Bennett ${ }^{\mathrm{a}, \mathrm{c}}$, B. Harper ${ }^{\mathrm{a}, \mathrm{c}}$, L. Lockyer ${ }^{\mathrm{a}, \mathrm{c}}$ and B. Powley ${ }^{\mathrm{a}}$

${ }^{\mathrm{a}}$ Telecommunications and Information Technology Research Institute, Australia; ${ }^{\mathrm{b}}$ School of Electrical, Computer and Telecommunications Engineering, Australia; ${ }^{\mathrm{c}}$ University of Wollongong, Australia

This paper proposes a system, the Smart Learning Design Framework, designed to support the development of pedagogically sound learning material within an integrated, platform-independent data structure. The system supports sharing, reuse and adaptation of learning material via a metadata-driven philosophy that enables the technicalities of the system to be imperceptible to the author and consumer. The system proposes the use of pedagogically focused metadata to support and guide the author and to adapt and deliver the content to the targeted consumer. A prototype of the proposed system, which provides proof of concept for the novel processes involved, has been developed. The paper describes the Smart Learning Design Framework and places it within the context of alternative learning object models and frameworks to highlight similarities, differences and advantages of the proposed system.

\section{Introduction}

Much of the current focus in the e-learning arena is centred upon the development of technical infrastructures that support reusability, interoperability, durability and accessibility of learning content (Bannan-Ritland et al., 2002; Welsch, 2002; Hummel et al., 2004). While much of this work has focused on the development of standards for learning objects, significant effort is also being made to develop standard descriptions for aggregations of learning objects; for example, in the form of a course module. The advantage of such an approach is that a module described using a standard specification could be transferred to any learning system able to interpret the standard,

*Corresponding author. Telecommunications and Information Technology Research Institute, University of Wollongong, Australia. Email: jasonl@elec.uow.edu.au 
thus enabling greater interoperability. However, considerable differences exist in the over-riding philosophies of proposed techniques.

The Sharable Content Object Reference Model (SCORM), which is becoming a de facto standard for delivering educational material (Olivier \& Liber, 2003), is based on the premise that learning content can be decomposed into discrete entities that are context independent. These 'content objects' are then 'combined with other instructional content objects, the aggregation provides the context and supports a defined learning experience' (Advanced Distributed Learning, 2004, pp. 1-24). While SCORM claims to be 'pedagogically neutral' (Learning Systems Architecture Laboratory, 2003, p. 1) and thus capable of supporting many different instructional designs, it is based on a content-driven, individual, self-paced approach to learning with a focus on tracking a learner's progress through the content (Kraan \& Wilson, 2002; Koper, 2003; Olivier \& Liber, 2003). Thus SCORM provides the technical infrastructure to facilitate the creation of reusable content, but does not prescribe or offer guidance to the author about how to produce effective content and sequence it in a way to produce pedagogically sound learning material (Pasini, 2004). This also implies that experts such as instructional designers are needed when determining what objects constitute a unit of learning. In fact, the SCORM documentation suggests that a development team is required to produce SCORM-compliant content (see Ip \& Canale, 2003, p. 672; Learning Systems Architecture Laboratory, 2003, pp. 13-14). Specifically, authors converting existing learning material to SCORM compliance must be able to structure the content in context-free objects, while those reusing existing SCORM objects must be able to aggregate those objects into meaningful learning sequences (Learning Systems Architecture Laboratory, 2003).

This approach may be suitable for large organisations that employ teams of specialist instructional designers, subject-matter experts, graphic designers and programmers, and that are concerned mainly with self-paced, individual training. However, it seems unlikely to transfer well to the higher education sector in which a much wider range of pedagogical approaches is used and teachers often design, develop and implement an e-learning course single-handedly or in small teams without specialist assistance.

As an alternative to SCORM, the IMS Learning Design (LD) specification (IMS Global Learning Consortium, 2003) promises to address at least one of these issues. IMS LD places learning activity at the fore by recognising that learning does not equate to content consumption (Tattersall \& Koper, 2003). It provides a language to describe a learning process, referred to as a 'unit of learning', in a standardised machine-readable format. IMS LD draws on Educational Modelling Language and is intended, unlike SCORM, to describe a broad spectrum of pedagogies with support for 'multiple learners as well as a single learner', 'behaviourist as well as constructivist approaches' and 'mixed mode (a mix of traditional and online learning, currently also referred to as "blended learning") as well as purely online learning' (Olivier \& Liber, 2003, p. 154). As such, IMS LD has the potential to describe a far greater array of learning process than SCORM. 
However, authors adopting the IMS LD specification must still know how to structure their educational material into pedagogically effective 'units of learning' to create lessons, modules or entire courses. Tools are emerging to make the design process easier as well as implementing the IMS LD specification; for example, Learning Activity Management System (Dalziel, 2003; LAMS, 2004) (based in part on IMS LD) and CopperCore. ${ }^{1}$ Although user-friendly authoring tools are an important step (Hummel et al., 2004), they may not be sufficient to support teachers to create effective e-learning. This issue is already being experienced in higher education, with many tertiary teachers facing significant challenges in developing effective online learning experiences, even with the use of learning management systems (Bennett \& Lockyer, 2004).

The work described in this paper aims to address the lack of pedagogical support inherent in existing systems by providing a platform-independent data structure encapsulated in an integrated pedagogically centred authoring process. The proposed Smart Learning Design Framework (SLDF) is underpinned by considerations about how higher education teachers would work with learning objects and the kinds of support they would need to create effective learning experiences. The SLDF utilises the MPEG-21 (Bormans \& Hill, 2002) standard for data structure, with author support implicit in the development of pedagogically sound content via the creation and use of meaningful metadata. The structure proposed for the SLDF supports content sharing, reuse, customisation and interoperability while integrating author support into a development process suitable for the single author in the higher education context. While SLDF offers a different perspective to the standard specifications already discussed, this does not preclude backward compatibility with these standards. In fact, while not presented with this emphasis in the following sections, SLDF could be viewed as an enhancement layer for SCORM and IMS LD, with translation back to these formats possible. However, this translation would necessarily result in some loss of SLDF functionality.

The paper is structured as follows. An overview of the proposed SLDF, in which its characteristics are highlighted and contrasted with alternative e-learning technical specifications, is presented in the next section. The overview is then expanded by providing a comprehensive description of the entire SLDF. The paper concludes by summarising the proposed SLDF technique and presents avenues of future research.

\section{The SLDF concept}

The SLDF proposed in this paper extends the framework presented in Lukasiak et al. (2005). The cornerstone of the SLDF is the seamless integration of a technical data structure with a well-supported process for developing pedagogically sound elearning materials.

The technical data structure is based on the MPEG-21 multimedia framework (Bormans \& Hill, 2002). MPEG-21 aims to provide a universal multimedia framework that permits the use of multimedia across a wide range of platforms and systems. The basic unit within the MPEG-21 framework is the Digital Item (DI). This is a 
'structured digital object with a standard representation, identification, and metadata' (Burnett et al., 2003). The DI is made up of three main components: resources, metadata, and structure. DIs represent the unit of transaction within the MPEG-21 standard and are universally represented using XML (Burnett et al., 2003).

Employing a broader, universal technical specification, as opposed to more specific learning technology specification (such as IMS), offers a number of advantages. These advantages include: the ability to exploit generic infrastructure (such as gateways and repositories) designed for the broader standard, thus promoting interoperability; the separation of content from presentation (as opposed to SCORM, which is HTML reliant), thus promoting reuse and customisation; and the ability to adapt content delivery based upon user preferences and content metadata, thus promoting adaptation. A detailed description of the application of MPEG-21 to e-learning materials is provided in Lukasiak et al. (2005).

The SLDF authoring process is based on the notion that a learning design can provide guidance for authors to create pedagogically sound learning material. A learning design refers to the way in which activities, resources and support mechanisms are planned and sequenced for students (Oliver \& Herrington, 2001). When described in a generic way, a learning design can serve as a framework or template into which learning objects and context-specific information can be incorporated (Bennett et al., 2004). Thus the learning design provides the pedagogical 'glue' to aggregate learning objects.

The outcome of the authoring process is referred to as a 'unit of study'. A unit of study may comprise a single activity, multiple activities, a lesson or module, or an entire subject or course. Thus, from a conceptual viewpoint, the SLDF would support authors in the following ways:

- Offer guidance in the selection and adaptation of a learning design suitable to the author's (teacher's) educational context.

- Assist with the selection and aggregation of suitable learning objects based on the chosen learning design.

- Package the unit of study for delivery to the learner(s).

The aggregation of learning objects into a unit of study is represented by a hierarchical structure in which learning objects are represented at the lowest level of the hierarchy. In the SLDF hierarchy, a learning object is defined as a digital resource that represents the smallest autonomous pedagogical unit that can be reused to support learning related to a specific purpose or intention (Agostinho et al., 2004; Lukasiak et al., 2005). Each learning object is represented as an MPEG-21 DI. This allows learning objects to have individual metadata and rights ${ }^{2}$ protection, and also facilitates the sharing and storage of the learning objects in large educational repositories that support the broader MPEG-21 format.

At the next level in the hierarchy, multiple learning objects can be aggregated to form a unit of study. The unit of study is also represented as an MPEG-21 DI, which embeds the learning objects (themselves individual digital items) as resources. A unit of study can also embed another unit of study, and so on. This format permits each 
unit of study to have unique metadata and rights embedded, while retaining the rights and metadata of the included learning objects and units of study. This hierarchical structure is represented in stages 1 and 2 of Figure 1, where the multiple MPEG-21 learning objects generated in stage 1 are embedded in the MPEG-21 unit of study of stage 2. A practical example of the hierarchical metadata structure and the retention of embedded metadata for individual learning objects within the SLDF is illustrated in a later section.

A significant feature of the SLDF is that the metadata schemas employed at the learning object and unit of study levels are different. The metadata schema at the learning object level is based on the IEEE LTSC Standard for Learning Object Metadata (IEEE LOM), and in terms of pedagogical information describes only the learning object's intended original use. Yet a teacher may use a learning object in a different way for different learning settings. Thus, another metadata schema, referred to as the 'Unit of Study Metadata', is used at the unit of study level and describes how the learning object is utilised within the context of the unit of study. This use of metadata is different to other hierarchical methods such as SCORM and IMS LD where the same metadata schema (based on IEEE LOM) is employed at each level of

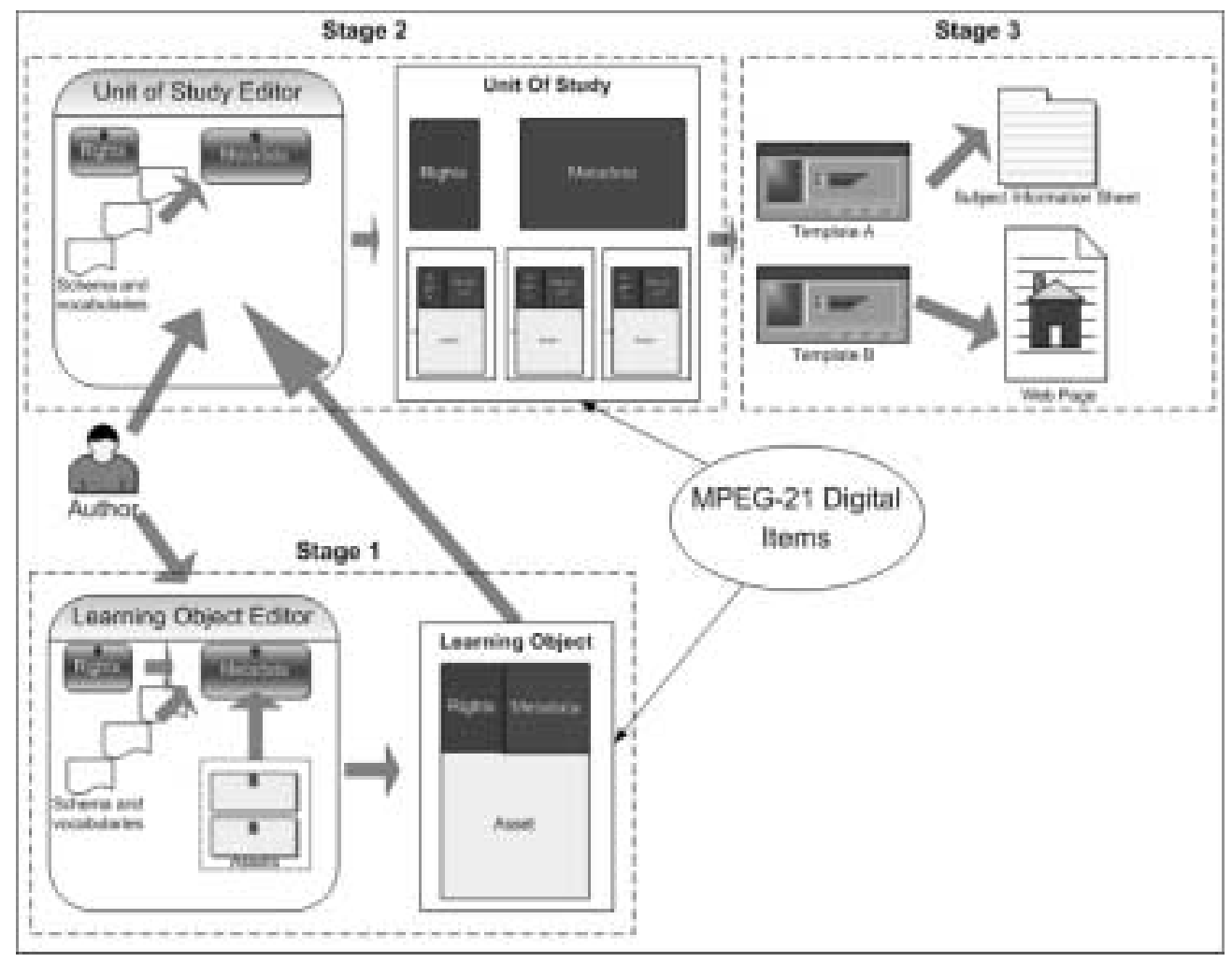

Figure 1. Overview of the SLDF process 
the hierarchy (IMS Global Learning Consortium, 2003; Advanced Distributed Learning, 2004).

Two editing tools work together seamlessly to support the SLDF authoring process as represented in Figure 1. At any time the author can work with either the Unit of Study (UOS) Editor or the Learning Object Editor.

The Learning Object Editor allows the author to select the pre-existing learning resources that will comprise the learning object. These are termed assets in Figure 1 and may include multiple files. The Learning Object Editor then assists the author to generate suitable metadata. This results in a learning object that complies with the SLDF application profile. Subsequently, and transparently to the author, the learning object editor creates an MPEG-21 DI that represents the object. The author can create metadata for learning objects prior to or during the development of a unit of study, or can import a learning object into the SLDF system that already has metadata associated with it.

The UOS Editor first assists the author select an appropriate learning design, and then guides him/her through the process of adding appropriate learning objects and contextual information specific to the unit of study. During this process the SLDF has the potential to provide the author with detailed assistance, including pedagogical guidance about the inclusion of appropriate objects, the design of activities and the choice of learning supports. The author assistance is based upon interrogation and analysis of the metadata embedded in learning objects, combined with pedagogical considerations specific to the learning design being employed. For example, it is envisaged that when an author selects a particular learning object to be included in the UOS, the UOS Editor examines the metadata associated with that learning object and prompts the author as to whether the selected learning object meets the pedagogical requirements of the learning design. However, as the embedded metadata describes the learning object's intended original usage, the system only prompts the author as to an apparent mismatch with the author able to ignore the notification if applicable. In this way the system makes meaningful use of the metadata but still allows the author to adapt the use of the learning object to his/her particular context. An example of how proof of concept for metadata based author assistance has been achieved for the prototype UOS Editor is detailed in a later section.

The interrogation and intelligent use of metadata implies that the generation and inclusion of appropriate metadata, at both the learning object and unit of study levels, is central to the SLDF concept. This metadata-driven approach requires the metadata employed at each stage to be unambiguous, reliable and descriptive. Thus, the metadata should mostly rely on well-structured schemas and vocabularies, and avoid large amounts of free text to ensure consistency of application. The approach taken to design the learning object and unit of study metadata schemes is detailed in later sections. The metadata-driven approach adopted in the SLDF contrasts with the content-driven approach adopted by systems such as SCORM. In these contentdriven approaches, metadata is included only for descriptive purposes and is often only useful in repository operations such as searching. 
While conceptually the Learning Object and UOS Editors are shown as different systems in Figure 1, practically they form part of an integrated software system with each editor differing primarily by the metadata schemas that are included. Common to both Learning Object and UOS stages is the fact that the output data structure takes the form of an MPEG-21 DI (Bormans \& Hill, 2002). This characteristic dictates that the unit of transaction in the SLDF is the MPEG-21 DI.

Using the MPEG-21 DI allows the SLDF to provide simple and seamless customisation of the content delivery. This is represented by the Delivery Stage (stage 3) in Figure 1, where templates are used to customise the content presentation. Customisation is possible because MPEG-21 separates content from its presentation (Bormans \& Hill, 2002) and the SLDF exploits this characteristic by using templates to extract the required content presentation from the UOS DI prior to final content delivery. A simple example application of the flexibility inherent in this system would be delivering similar learning content to students enrolled in the same subject at different locations. Employing the SLDF would allow an institution to generate a single SLDF UOS and customise the templates to meet the requirements for the different locations. More sophisticated customisations are also possible; for example, to students using different display devices or to suit different modes of teaching.

The subsequent three sections of this paper expand on the overview already presented and provide detailed explanation and discussion of each stage in Figure 1.

\section{Learning Objects in the SLDF}

\section{Description and structure}

As described in the previous section, the SLDF software systems include a Learning Object Editor, which allows the seamless generation of MPEG-21 DIs that represent the learning object and its metadata and rights. This tool promotes the inclusion of pedagogically comprehensive and repeatable metadata via pull-down menus generated from an XML-based metadata schema. The metadata schema has been designed to restrict the use of free text and to instead employ fixed vocabularies for many of the metadata fields (see next section for details of the metadata development). Structuring the schema to minimise free text promotes metadata repeatability and facilitates machine-based operations such as efficient object retrieval from repositories, automated adaptation of objects to meet requirements and intelligent author assistance when embedding objects into units of study. Also, utilising an XML-based schema to generate the pull-down menu fields allows the tool to be simply modified, expanded or customised via the production of new metadata schemas or the inclusion of alternative metadata schemas.

The Learning Object Editor has also been designed to make the technical complexities of the system imperceptible to the author, by automatically generating conforming MPEG-21 DIs behind the scenes. The tool also parses the data via conforming XML schemas to generate the DIs. This allows the tool to be easily customised to 
generate alternative data structures (such as SCORM) through the use of alternative XML schema files for data content.

\section{Learning Object metadata for higher education}

The SLDF is premised on the notion that Learning Objects are resources that, if appropriately annotated with metadata, can be retrieved easily and incorporated into a learning design. There has been considerable interest in the development of metadata schemas over the past decade, with two main initiatives being The Dublin Core Metadata Initiative ${ }^{3}$ and the IEEE LTSC Standard for Learning Object Metadata (IEEE, 2002). The learning object literature highlights that these metadata schemas, particularly IEEE LOM, are being customised to meet the specific needs of communities. The use of metadata application profiles-where communities annotate their learning objects in more specific ways, yet adhere to a metadata standard's overall structure (e.g. incorporating specific vocabularies for certain metadata elements, and deciding which metadata elements are mandatory and optional)—is becoming prevalent. Thus, IEEE LOM was closely examined by reviewing critiques of LOM, directly applying the Educational category descriptors to a number of Learning Objects, and then examining a well-researched LOM-based application profile. The application profile devised incorporates recommendations from CanCore (Friesen et al., 2003) and includes specific vocabularies suitable for the Australian Higher Education context. A detailed description of the development of the application profile for higher education can be found in Agostinho et al. (2004). ${ }^{4}$

\section{Example}

The screen capture shown in Figure 2 shows an image of a girl walking with ball in hand. The image is annotated with a directional arrow and the symbol $\mathrm{X}$ placed directly above a cup, which sits on the ground. The image is accompanied by text describing the girl as walking at a constant pace with ball in her hand. This image can be considered a learning object because it has been created in this form specifically for instructional purposes.

A screen capture of the Learning Object Editor in Figure 3 shows the metadata record being created for this learning object by the author.

The Editor automatically generates fields such as resource location, MIME type and size from the technical specifications of the selected file(s). Automated completion of the title field is only treated as a suggestion and the author is free to replace the automated value with a more descriptive title. The author's details can be stored as a user profile in the application preferences and are automatically included in the Contributors field, although this data can be altered if necessary. The majority of the remaining fields are specified by selecting the appropriate term from pull-down menus, with free text limited to fields such as Title, Description, Keywords and Educational Use, and to specify usage restriction details. The author can access guidance about how to complete each metadata field by clicking on the question mark icon associated with each field. 


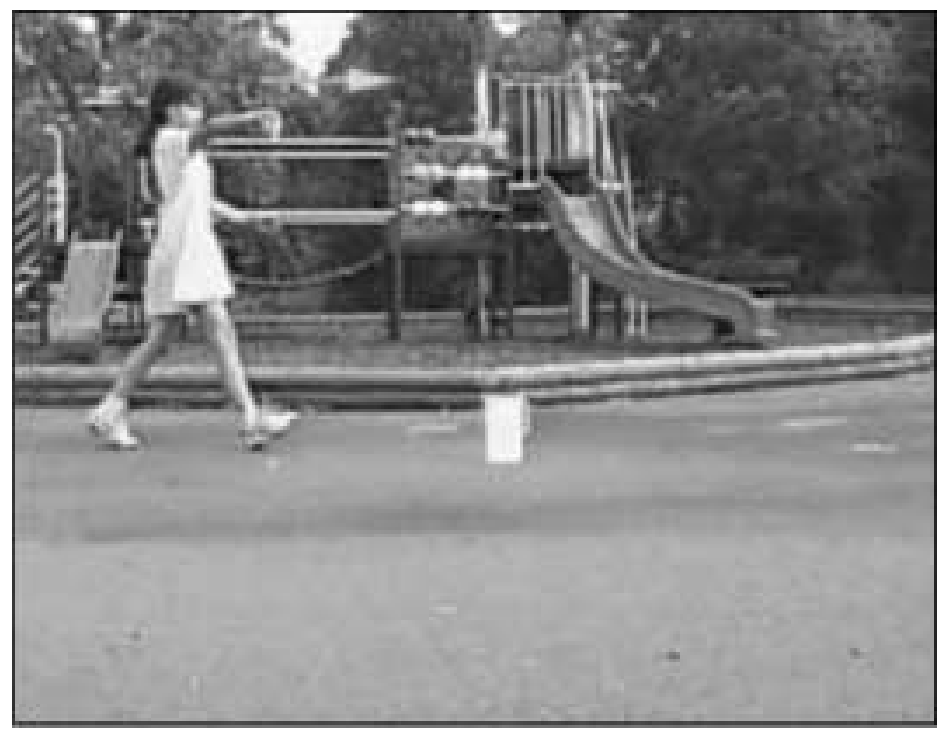

Figure 2. Image of girl with a ball (Kearney \& Wright, 2002)

The application profile has been designed to provide detailed pedagogical information that can be used to inform the selection of learning objects when embedding them into a UOS. This is achieved through the use of controlled vocabularies for the LOM elements 5.2 Learning Resource Type, 5.6 Context, and 5.7 Typical Age Range. These fields are represented in Figure 3 as, respectively: Type of learning resource (diagram, image, scenario); Context of Use (university undergraduate); and Audience status/age (first-year undergraduate). In specifying the type of learning resource for a Learning Object, the author selects appropriate values from the IEEE LOM vocabulary and then chooses further values from an additional vocabulary specific to higher education developed for this project. This additional vocabulary provides a richer set of options that allows the author to clearly describe the form and function of the object. The contrast between this additional vocabulary and the available LOM vocabulary for learning resource type is evident in Figure 3 by examining the LOM and SLDF entries in the type of learning resource field. This example clearly demonstrates the improved pedagogical description available via the inclusion of the SLDF vocabulary.

\section{Unit of study in the SLDF}

\section{Concept and overview}

The SLDF structure presents a seamless mechanism whereby learning objects and other units of study may be grouped together into a single unit of study, while still retaining the rights, metadata and functionality of the individual objects. This retention of rights, metadata and functionality at each level creates a clear hierarchical 


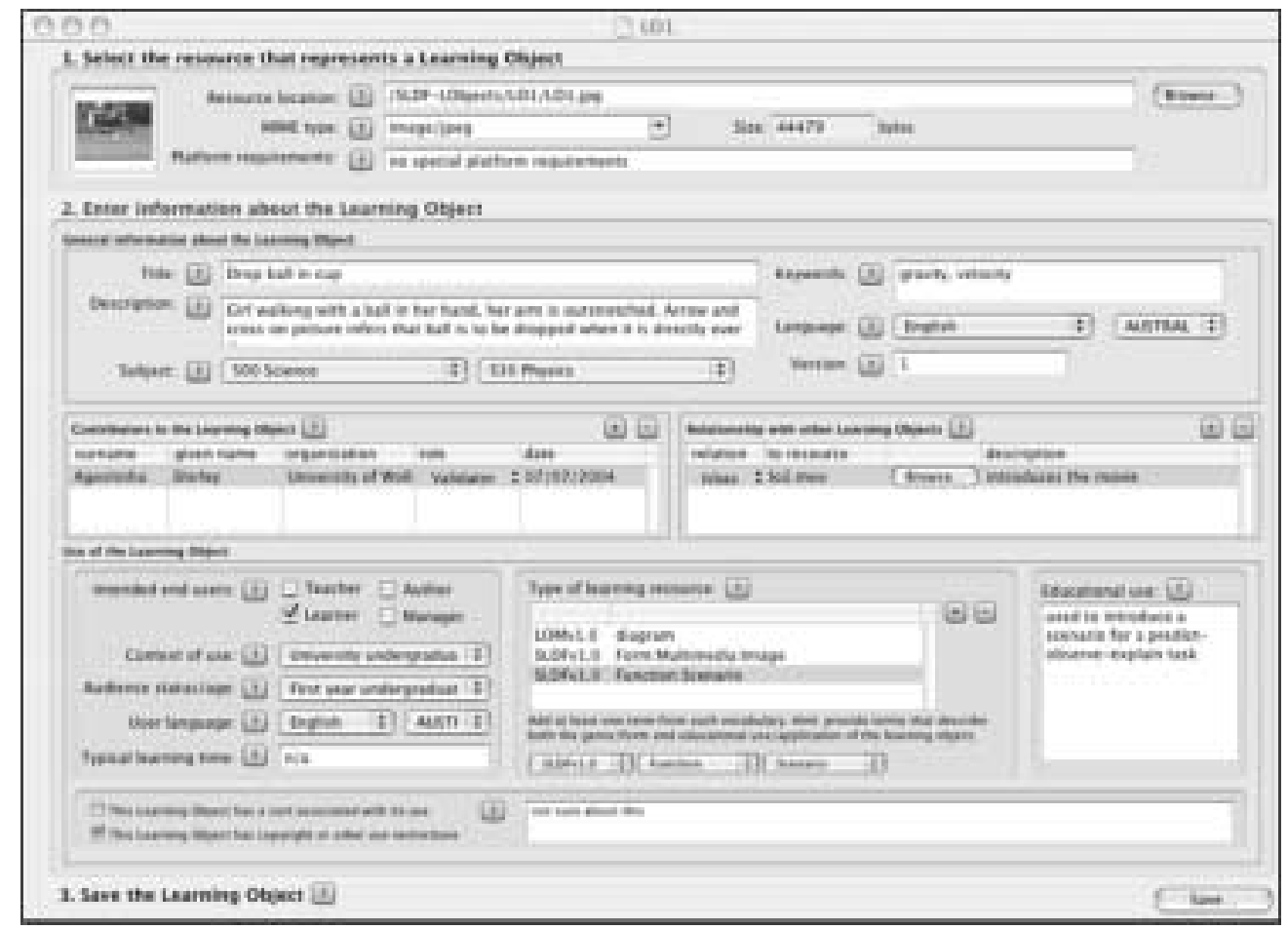

Figure 3. Creating a metadata record for the image of a girl with a ball

structure within which the complexity of the objects/units of study increase as the level of the hierarchy increases. Also, the metadata becomes less objective (databased) and more subjective (context-based) as one progresses up the hierarchy. The unit of study metadata describes the pedagogical approach and sequence in which the embedded content is to be consumed, while learning object metadata (see previous section) describes the specific content of the associated data.

\section{UOS metadata}

There are calls from educators for richer metadata to describe how learning objects are used to create a learning experience; that is, how they are sequenced, aggregated and combined (Jonassen \& Churchill, 2004). Current metadata initiatives are focused at the learning object level and there are no metadata schemas that describe how learning objects are aggregated and used in a learning environment. As introduced earlier, the SLDF proposes to address this problem by employing different (pedagogically distinct) metadata schemas at the Learning Object and UOS levels.

The SLDF UOS metadata provide information about the activity students are to perform and how the learning objects will be used to support them. The purpose of the UOS metadata is twofold. It can be used in machine-readable format to inform 
the delivery of the unit of study, and can also be used to generate documentation for the educator about the UOS in the form of a summary report/course outline. This functionality contrasts with the IMS LD specification, which provides a language to describe a 'unit of learning', which is machine-readable and can be interpreted by an IMS LD aware player (Hummel et al., 2004). Unlike the UOS metadata, it is not intended that IMS LD be interpreted by the educator.

The UOS metadata schema has been designed to describe the tasks, resources and the supports to be applied with the chosen learning design: the tasks being what students are required to do in the learning environment, the resources being provided to help students complete the tasks, and supports defined as the mechanisms to help the students complete the tasks (Oliver \& Herrington, 2001). The organisational structure of the UOS metadata schema is similar to IEEE LOM, but the fields within the categories have been tailored to provide description more relevant to the UOS, particularly for the Educational category.

\section{Example}

At present, proof of concept for the SLDF unit of study functionality has been achieved via the inclusion of a Predict-Observe-Explain (POE) learning design (Kearney \& Wright, 2002) into the UOS Editor. It is envisaged that future enhancements will allow multiple generic learning designs to be available via a pull-down menu in the UOS Editor. The following demonstrates an example of the SLDF system applied to the POE learning design. The generic version of the POE learning design is graphically represented in Figure 4.

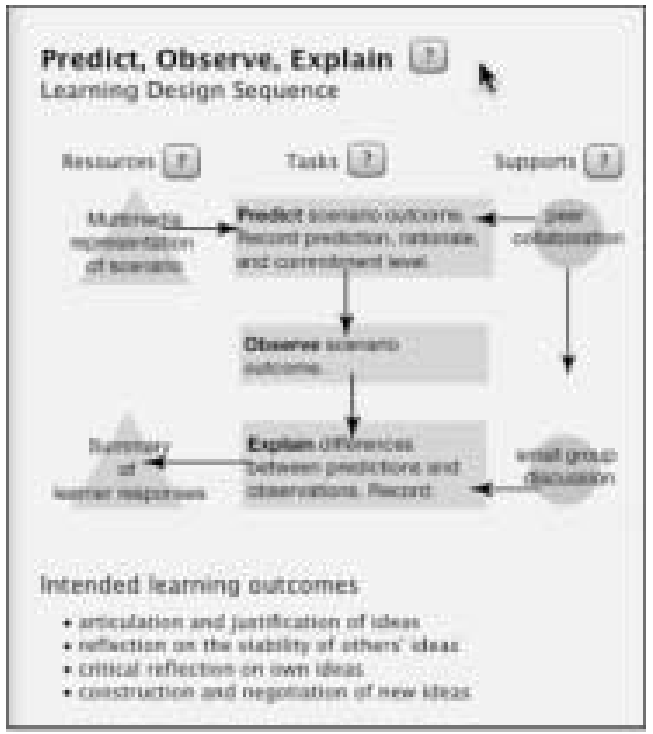

Figure 4. POE Learning Design 
The SLDF UOS Editor allows the author to specify learning objects and contextually specific information for a UOS based on this framework. As with the Learning Object Editor, the author can access detailed assistance by clicking on the questionmark buttons. For example, general information about the POE learning design (i.e. what tasks students are required to conduct, what resources are required to help with the tasks and how students should be supported) is available by clicking on the question marks in Figure 4. A screen capture of the interface to develop a POE unit of study is shown in Figure 5.

Detailed pedagogical guidance - such as how to select an appropriate scenario, what learning objects to include, how the prediction question and multiple-choice options should be structured-is provided, again via clicking on the question-mark buttons. An example of this pedagogical support is illustrated in Figure 6.

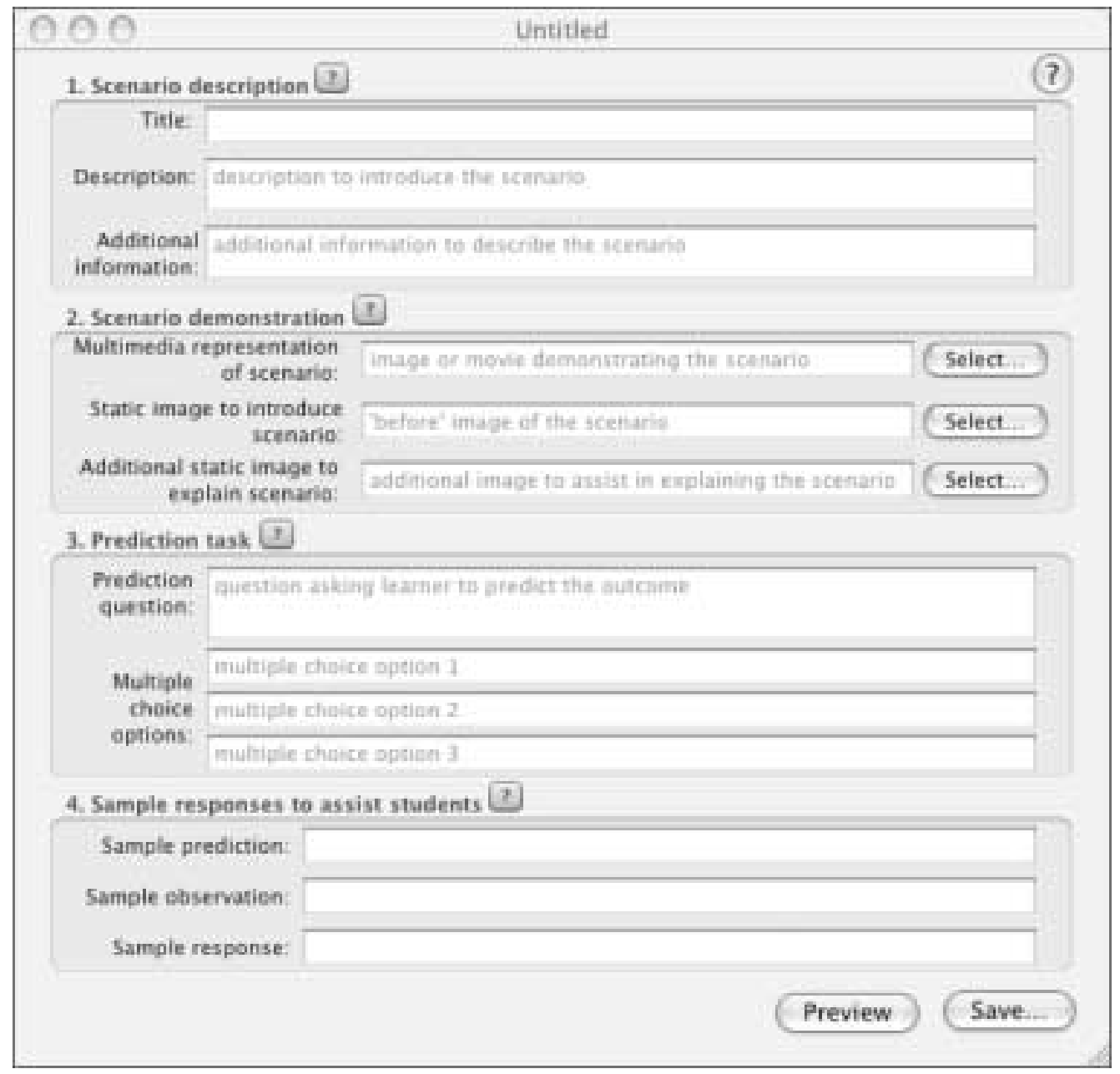

Figure 5. Screen capture of POE Learning Design interface in UOS Editor 


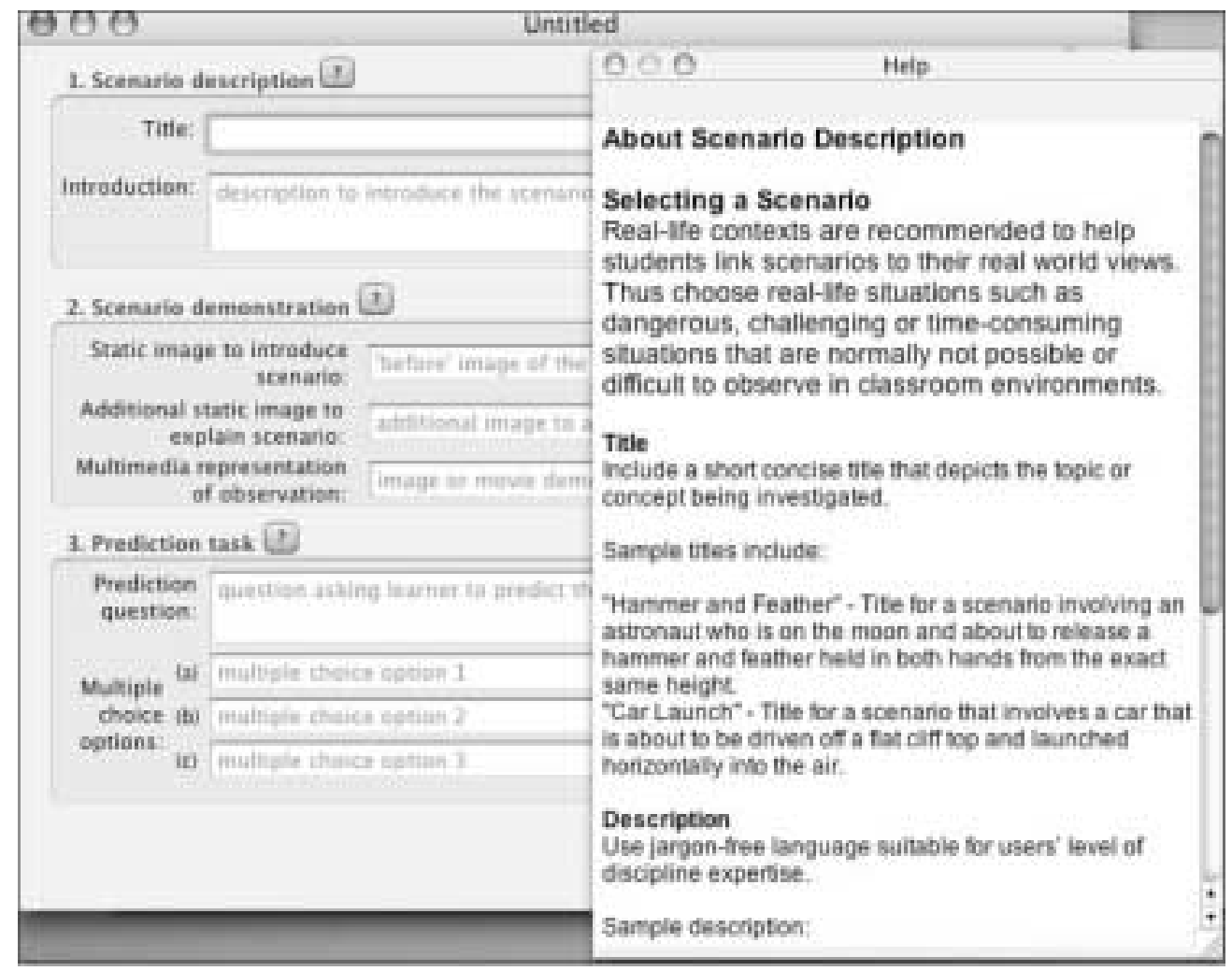

Figure6. ExampleofpedagogicalsupportprovidedbytheUOSEditorwhendesigningaPOEUOS

When a generic learning design is chosen, the UOS metadata record is created and some values can be automatically generated. These generic values can be used to support the authoring process. For example, the learning design detailed in Figure 4 requires that a multimedia representation of a scenario be included. Thus, when a learning object is selected for inclusion, the embedded learning object metadata is interrogated. If the learning object shown in Figure 3 is selected for inclusion in the POE UOS, the values associated with 5.2 Learning Resource Type are checked to see if they match the pedagogical requirements of the learning design (in this instance, the prototype checks that the learning resource type defines a multimedia representation, as required for the POE learning design). If there is a mismatch, the author is notified and can decide whether to include the learning object or select another.

Table 1 presents the form of the UOS metadata. The table includes the educational descriptors, with example values specified for the prediction task, which uses the image resource shown in Figure 2 and support for peer discussion. Controlled vocabularies are used to accompany the use of free text fields. In this way, the UOS metadata can be interrogated both by a teacher and the system. Work is currently underway to refine the UOS metadata schema. 


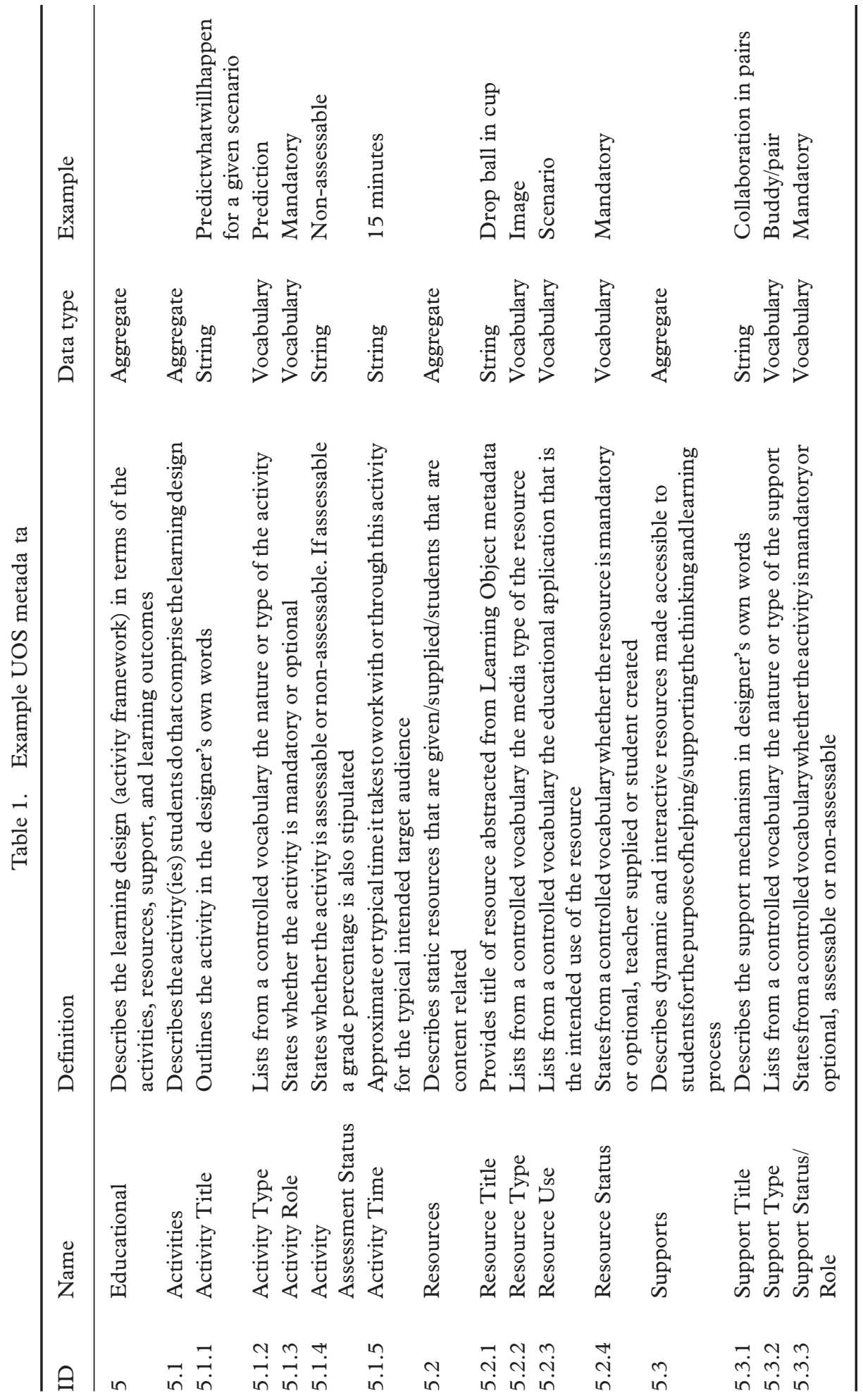




\section{Delivery of UOSs}

Traditionally, e-learning content has been delivered via HTML in the form of webpages. This is certainly the constraint of systems such as SCORM (Advanced Distributed Learning, 2004) in that employing HTML as the data structure introduces an implicit limitation that content and presentation are inseparably linked. This linkage of content and presentation introduces considerable restriction when customising the presentation of content for various audiences. The SLDF removes this constraint by operating within the MPEG-21 standard, thus the unit of transaction within the SLDF is the MPEG-21 DI. MPEG-21 employs an XML-based data format that has been intentionally selected to separate the data content from its presentation.

The SLDF supports explicit flexibility in content presentation via the application of templates to the UOS at the point of delivery. Due to their versatility and universal format, XSLT style sheets have been chosen as the format for representing the SLDF templates. The process of flexibly presenting an SLDF unit of study, represented in stage 3 of Figure 1, shows the same UOS being used to generate different presentation formats via the application of different templates. The prototype UOS currently supports templates that permit delivery of the POE unit of study as either a webpage or a subject overview/handout. It is the vision of the SLDF that a range of templates for each learning design be available within the UOS Editor. These templates will support common standard formats, such as HTML and PDF. It is envisaged that authors will be able to customise the presentation of their content by editing the standard templates, producing their own templates or importing existing templates.

Figure 7 represents a possible scenario in which the example UOS for a higher education course can be presented in two distinct formats. The right-hand presentation represents a text-based handout, while the left-hand presentation constitutes a website that delivers course content such as images, video and learner interaction via form fields. In this example, the SLDF uses the unit of study to produce both instances using appropriate templates to extract the data into the required presentation format. The distinct advantage is that the author only produces a single unit of study. Traditionally, such an example would require the author to generate the two forms of learning content themselves.

While not readily apparent in Figure 7, there is also inherent flexibility in the delivery method for SLDF content. For example, the template extraction process could be server based, user based or performed prior to transmission. In a server-based scenario, the UOS DI would be transmitted directly to an MPEG-21-compliant server. The remote server would then process the unit of study and deliver the appropriate presentation to the end users, based on student and system metadata (or preferences). For example, a student accessing the course over a handheld computer may not have the infrastructure necessary to display the full HTML presentation. In this situation the MPEG-21-compliant server could detect the display device via user descriptors and deliver only a visually restricted version of the unit, such as a textbased subject information sheet. This scenario provides a practical example of the DI adaptation philosophy detailed in Vetro et al. (2002) and Vetro and Timmerer 


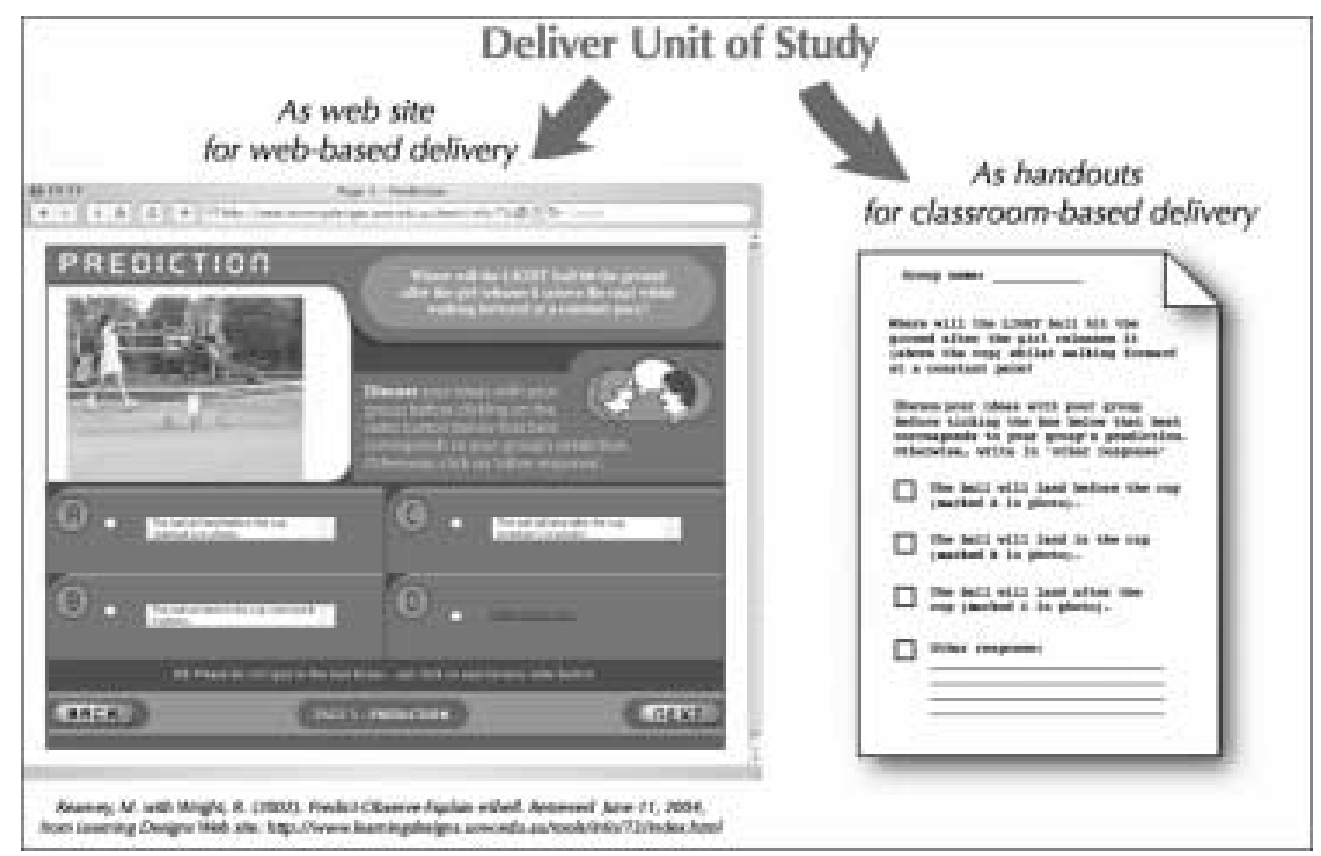

Figure 7. Example of adaptive content presentation

(2005), and presents another potential advantage of using a universal technical specification for delivering learning content.

A user-based scenario would see the unit of study DI being delivered directly to all end users. The users could then apply their own templates to extract the presentation that they currently require. Alternatively, the required presentation could be centrally extracted from the unit of study DI prior to any transmission and then delivered directly to the remote users via a company intranet.

An alternative to producing a customised presentation from the SLDF unit of study via a template is to simply use any MPEG-21-compliant browser to view the contents. Currently, a free MPEG-21 browser is available from Enikos. ${ }^{5}$ While employing a universal MPEG-21 browser to view the unit of study would limit the functionality and adaptability of the presentation, it does signify another advantage of working within a universal technical standard, in that universal players and browsers should be freely available.

\section{Conclusions and further work}

The proposed SLDF represents an integrated system developed to support the transparent authoring of pedagogically sound educational material. The system addresses issues of platform independence, data transparency and adaptation by operating 
within the MPEG-21 framework. In addition, object sharing, reuse and repeatability are addressed through the inclusion of well-defined metadata.

As opposed to other systems, such as SCORM, the proposed system is centred on developing pedagogically sound learning material. Pedagogical support is provided via the integration of learning designs and pedagogically descriptive metadata into the authoring process. A novel hierarchy of metadata is utilised whereby UOSs, which are highly context focused, are described with metadata different to learning objects, which have far less context and are more content orientated.

The primary advantages of the SLDF system can be summarised as follows:

- Guidance and support for the development of appropriate learning context and pedagogy is implicit in the system.

- Assistance with the selection and aggregation of suitable learning objects based on the chosen learning design is possible via interrogation of the metadata included in the selected learning objects.

- It represents an integrated metadata-driven system where pedagogical soundness and technical transparency are implicit.

- Technical complexities such as data structure and programming are transparent to the author. At no stage in the process is the author aware (or required to be aware) of the fact that the learning material being developed is packaged as data units conforming to the MPEG-21 standard.

- Learning objects are assembled into digital items that exhibit a transparent data format and facilitate storage, adaptation and reuse (i.e. use in online repositories).

- Meaningful metadata (description) is included at both the Learning Object and UOS levels. This metadata supports both reuse by other authors and provides objective targets for adaptation and customised delivery.

- Data are separated from presentation, which in turn supports seamless and simple customisation of presentation via templates.

- It scales from a complex design paradigm employing educational designers, instructional designers, artists and programmers to a single author in an unsupported environment such as higher education.

Current work involves a practical evaluation of the usability and usefulness of the SLDF in a real educational setting. This work involves a test group of 12 secondary teachers. Avenues for future work include: refinement of the metadata schemas utilised at both Learning Object and UOS levels; development of repositories suitable for storing, searching and retrieving the proposed learning objects; refinement of the template process used for content presentation; integration of the proposed system into existing learning management systems; and compatibility with existing educational technology standards.

\section{Acknowledgements}

The authors would like to acknowledge the support of the Smart Internet Technology Cooperative Research Centre (http://www.smartinternet.com.au) that has funded 
this project: A Smart Learning Design Framework (Project Team: Barry Harper, Sue Bennett, Lori Lockyer, Jason Lukasiak, Shirley Agostinho, and Brett Powley). The team also thanks Ian Burnett and the MPEG-21 company Enikos for assistance with the MPEG-21 standard. The SLDF team would also like to thank Matthew Kearney from University of Technology Sydney for his assistance in implementing the Predict-Observe-Explain learning design.

\section{Notes}

1. See http://coppercore.org/

2. The generation and inclusion of digital rights is beyond the scope of this paper. The digital rights management section of MPEG-21 is currently being developed and additional information is provided in ISO/IEC 21000-6:2004 (2004) and Wang et al. (2005).

3. See http://dublincore.org/

4. The application profile is available online (http://www.digitalmedia.uow.edu.au/sldf.html).

5. See http://www.enikos.com/home.shtml

\section{References}

Advanced Distributed Learning (2004, July 22) Sharable Content Object Reference Model (SCORM ${ }^{\circledR}$ ) 2004 2nd edition overview. Advanced Distributed Learning. Available online at: http://www.adlnet.org/screens/shares/dsp_displayfile.cfm?fileid=994 (accessed 4 October 2004).

Agostinho, S., Bennett, S., Lockyer, L. \& Harper, B. (2004) Developing a learning object metadata application profile based on LOM suitable for the Australian Higher education context, Australian fournal of Educational Technology, 20(2), 191-208.

Bannan-Ritland, B., Dabbagh, N. \& Murphy, K. (2002) Learning object systems as constructivist learning environments: related assumptions, theories, and applications, in: D. A. Wiley (Ed.) The instructional use of learning objects (Bloomington, IN, Agency for Instructional Technology and Association for Educational Communications \& Technology), 6197.

Bennett, S. \& Lockyer, L. (2004) Becoming an online teacher: adapting to a changed environment for teaching and learning in higher education, Educational Media International, 41(3), 231-244.

Bennett, S., Lockyer, L. \& Agostinho, S. (2004) Investigating how learning designs can be used as a framework to incorporate learning objects, in: R. Atkinson, C. McBeath, D. JonasDwyer \& R. Phillips (Eds) Beyond the comfort zone: Proceedings of the 21st Annual Conference of the Australasian Society for Computers in Learning in Tertiary Education, Perth, 5-8 December, 116-122. Available online at: http://www.ascilite.org.au/conferences/perth04/procs/ bennett.html (accessed 31 January 2005).

Bormans, J. \& Hill, K. (Eds) (2002) MPEG-21 overview v.5. International Organisation for Standardisation, Organisation Internationale De Normalisation ISO/IEC JTC1/SC29/ WG11 Coding of Moving Pictures and Audio. Available online at: http://www.chiariglione.org/mpeg/standards/mpeg-21/mpeg-21.htm (accessed 22 May 2003).

Burnett, I., Van de Walle, R., Hill, K., Bormans, J. \& Pereira, F. (2003) MPEG-21: goals and achievements, IEEE transactions on Multimedia, 10(4), 60-70.

Dalziel, J. (2003) Implementing learning design: the learning activity management system (LAMS), in: G. Crisp, D. Thiele, I. Scholten, S. Barker \& J. Baron (Eds) Interact, integrate, impact, Proceedings of the 20th Annual Conference of the Australasian Society for Computers in Learning in Tertiary Education, 7-10 December (Adelaide, SA, ASCILITE), 593-596. 
Friesen, N., Fisher, S. \& Roberts, A. (2003, September 10) CanCore learning resource metadata application profile: CanCore guidelines for the implementation of learning object metadata version 1.9. Athabasca University. Available online at: http://www.cancore.ca/ (accessed 11 October 2003).

Hummel, H., Manderveld, J., Tattersall, C. \& Koper, R. (2004) Educational modelling language and learning design: new opportunities for instructional reusability and personalised learning, International fournal of Learning Technology, 1(1), 111-126.

IEEE. (2002, July 15). Draft standard for learning objects metadata. Learning Technology Standards Committee of the IEEE. Available online at: http://tsc.ieee.org/doc/wg12/ LOM_1484_12_1_v1_Final_Draft.pdf (accessed 13 March 2003).

Ip, A. \& Canale, R. (2003) Supporting collaborative learning activities with SCORM, paper presented at the Proceedings of the EDUCAUSE in Australasia 2003 Conference, University of Adelaide, 6-9 May.

IMS Global Learning Consortium. (2003) IMS learning design best practice and implementation guide version 1.0 final specification. IMS Global Learning Consortium Inc. Available online at: http://www.imsglobal.org (accessed 11 October 2003).

ISO/IEC 21000-6:2004. (2004) Information technology—multimedia framework (MPEG-21)—part 6: rights expression language (International Standards Organisation, Geneva).

Jonassen, D. \& Churchill, D. (2004) Is there a learning orientation in learning objects?, International fournal on E-Learning. April-June, 32-41.

Kearney, M. \& Wright, R. (2002) Predict-observe-explain eshell. Learning Designs. Available online at: http://www.learningdesigns.uow.edu.au/tools/info/T3/index.html (accessed 23 July 2004).

Koper, R. (2003) Combining reusable learning resources and services with pedagogical purposeful units of learning, in: A. Littlejohn (Ed.) Reusing online resources: a sustainable approach to e-learning (London, Kogan Page), 46-59.

Kraan, W. \& Wilson, S. (2002, October 2) Dan Rehak: 'SCORM is not for everyone'. Center for Educational Technology and Information Systems. Available online at: http:// www.cetis.ac.uk/content/20021002000737 (accessed 28 June 2004).

LAMS (2004) Learning Activity Management System. Available online at: http://www.lamsinternational.com/ (accessed 1 February 2004).

Learning Systems Architecture Laboratory (2003) SCORM best practices guide for content developers. Carnegie Mellon University. Available online at: http://www.lsal.cmu.edu/lsal/expertise/ projects/developersguide/developersguide/guide-v1p0-20030228.pdf (accessed $22 \mathrm{March}$ 2004).

Lukasiak, J., Agostinho, S., Burnett, I., Drury, G., Goodes, J., Bennett, S., Lockyer, L. \& Harper, B. (2005) A framework for the flexible content packaging of learning objects and learning designs, Fournal of Educational Multimedia and Hypermedia, 13(4), 465-481.

Oliver, R. \& Herrington, J. (2001) Teaching and learning online: a beginner's guide to e-learning and e-teaching in higher education (Edith Cowan University, Mt Lawley, Western Australia).

Olivier, B. \& Liber, O. (2003) Learning content interoperability standards, in: A. Littlejohn (Ed.) Reusing online resources: a sustainable approach to e-learning (London, Kogan Page), 146155.

Pasini, N. (2004) The role of SCORM in e-learning. Learning Systems Architecture Laboratory, Carnegie Mellon University. Available online at: http://www.lsal.cmu.edu/lsal/expertise/ papers/notes/scormrole20040119/scormrole-v1p0-20040119.html (accessed 21 June 2004).

Tattersall, C. \& Koper, R. (2003) EML and IMS learning design: from LO to LA. Educational Technology Expertise Centre, The Open University of the Netherlands. Available online at: http://www.ltsn.ac.uk/genericcentre/index.asp?id=18742 (accessed 14 January 2004).

Vetro A. \& Timmerer, C. (2005) Digital item adaption: overview of standardization and research activities, IEEE Transactions on Multimedia, 7(3). 
Vetro, A., Perkis, A. \& Devillers, S. (Eds) (2002) MPEG-21 digital item adaptation WD (version 2.0). ISO/IEC JTC1/SC29/WG11/N4944 (Klagenfurt).

Wang X., De Martini T., Wragg B. \& Paramasivam M. (2005) The MPEG-21 rights expression language, IEEE Transactions on Multimedia, 7(3).

Welsch, E. (2002) SCORM: clarity or calamity?, Online Learning, 6(6). Available online at: http://www.onlinelearningmag.com/onlinelearning/magazine/ article_display.jsp?vnu_content_id=1526769 (accessed 21 June 2004). 